\title{
Aging does not affect spermatogenic recovery after experimentally induced injury in mice
}

\author{
Jens Ehmcke, Bhavika Joshi, Scott D Hergenrother and Stefan Schlatt \\ Department of Cell Biology and Physiology, University of Pittsburgh School of Medicine, W952 Biomedical Science \\ Towers, 3500 Terrace Street, Pittsburgh 15261, Pennsylvania, USA
}

Correspondence should be addressed to S Schlatt; Email: schlatt@pitt.edu

\begin{abstract}
Testes in aging mammals undergo a variety of age-related changes, such as reduction of size, lower sperm output, an increase in abnormal forms of sperm, and endocrine malfunctions. It has been suggested that the spermatogenic defects are due to loss and dysfunction of spermatogonial stem cells as well as deterioration of the tubule microenvironment. In the present study, we explore the depletion and recovery of spermatogenesis in young ( 3 month) and old (12 month) mice exposed to cooling, X-irradiation ( 5 Gy) or cytotoxic treatment using Busulfan $(40 \mathrm{mg} / \mathrm{kg})$. We aim to determine a potential age-related change of vulnerability to gonadotoxic treatments by describing the intensity of spermatogenic depletion and the degree of spermatogenic recolonization with qualitative and quantitative parameters on organ weights and histological parameters at two time points ( 2 weeks, depletion; 6 weeks, recovery). Our data reveal specific acute effects of cooling on multinucleation of germ cells but no other severe injury. Irradiation and Busulfan-treatment exerted the expected depletional wave of germ cells leading to severe testicular injury and spermatogenic failure. The recovery of spermatogenesis occurred in both treatment groups and both age groups to a similar extent. We therefore noted no prominent age-related differences in spermatogenic depletion and recovery in any treatment group. We conclude that in both age groups, the remaining spermatogonial stem cells are capable to induce spermatogenic recovery and the aging tubule microenvironment at $\mathbf{1}$ year has not become more vulnerable to irradiation, Busulfan-treatment or testicular cooling.

Reproduction (2007) 133 75-83
\end{abstract}

\section{Introduction}

Stem spermatogonia represent the starting point for spermatogenesis. Their well-controlled renewal and generation of differentiating progeny is needed to generate millions of sperm per day (Ehmcke et al. 2006). A depletion of spermatogonial stem cells is the apparent cause for infertility in oncological patients after chemo- and radiotherapy (Schlatt et al. 2000, Radford 2003, Wallace \& Thompson 2003), and might play a leading role in the oligo- and azoospermia of infertility patients (Takagi et al. 2001, Yakirevich et al. 2003). Meiotic and post-meiotic germ cells (spermatocytes and especially the spermatids) are much less sensitive to cytotoxic treatment and radiation when compared with spermatogonia (Meistrich et al. 1978).

Although chemo- and radiotherapy are applied to patients of all ages, no study has yet addressed the differences in the impact of these therapies on the reproductive potential of younger versus older adult gonads. The testicular function in aging men appears only marginally affected in contrast to aging women, who lose their fertility potential due to the depletion of the primordial follicle pool (Gosden \& Faddy 1994, Fauser 2000, Skinner 2005), but the severity of defects in the aging human testis is controversial. Some reports show a constant and slow decline of serum androgen levels (Nieschlag et al. 1982, Neaves et al. 1984) but no decrease in sperm parameters (Nieschlag et al. 1982, Neaves et al. 1984, Johnson 1989, Haidl et al. 1996, Eskenazi et al. 2003). In contrast, other studies indicate lower sperm concentrations, lower total semen volume and a higher index of morphologically abnormal sperm in older men (Pasqualotto et al. 2005) and a higher index of arrested germ cell divisions (Miething 2005). A drop in sperm production was related at least in part to an increased rate of apoptosis in germ cells (Brinkworth et al. 1997, Kimura et al. 2003). Several age-related histological changes of the seminiferous parenchyma have been described (Honore 1978, Johnson et al. 1986, Paniagua et al. 1987, de Miguel et al. 1997, Plas et al. 2000, Pal \& Santoro 2003). 
In mice, testis size starts decreasing at 12 months of age and Sertoli cell-only tubules become more abundant (Ryu et al. 2006). The number of tubules with normal spermatogenesis starts decreasing and the degree of fibrosis in the testis increases (Wirth-Dzieciolowska \& Czuminska 2000, Zhang et al. 2006). In addition, Suzuki \& Withers (1978) state that the number of spermatogonial stem cells in mice decreases exponentially with age. Thus, although most mouse strains live well beyond 1 year of age, male mice show first signs of reproductive aging at 1 year of age. In contrast, studies by Ryu et al. (2006) indicate that mouse spermatogonial stem cells do not accumulate age-related defects. We selected mice of 3 months and 1 year as our two age groups since we aimed to explore whether spermatogenesis is already vulnerable in the normally functioning 1 -year old testis when compared with the fully active 3 -month old testis. It seemed appropriate to not select an old group which already showed severe signs of age-related deterioration in a number of organs as this would have complicated the analysis and interpretation of the anticipated effects.

The three gonadotoxic challenges to the testes, employed to study the vulnerability to injury in young and old, were testicular cooling, X-irradiation and Busulfan-exposure. The cellular effects of both X-irradiation and Busulfan-treatment are well known and characterized (van Keulen \& deRooij 1975, Meistrich et al. 1978), targeting primarily the spermatogonial stem cells and rapidly dividing pre-meiotic germ cells. The effects of testicular cooling are not well characterized, but this strategy has been used to deplete germ cells from rat testes (Zhang et al. 2004). Sampling animals from all age and treatment groups at an early time-point to detect germ cell depletion and at a later time-point to explore spermatogenic recovery allows us to determine any age-related difference to the experimentally induced challenge. In addition, the analysis of the androgen status revealed age-related changes of endocrine testicular function.

\section{Materials and Methods}

\section{Animals}

Sixty young adult male BALB/C mice were purchased from Harlan (Indianapolis, IN, USA). At 3 months of age, the animals were included in the study. They are referred to as 'young mice' in the following. Sixty-five old male $\mathrm{BALB} / \mathrm{c}$ mice were purchased from the National Institute on Aging (NIA; Bethesda, MD, USA). At 12 months of age, these mice were included in the study. They are referred to as 'old mice' in the following. As indicated earlier, testicular changes can be detected already at 1 year of age in most mouse strains. The number of tubules with normal spermatogenesis decreases and the degree of fibrosis in the testis increases already at 1 year of age in different strains of mice (Wirth-Dzieciolowska \&
Czuminska 2000, Zhang et al. 2006). In order to study age-related changes of spermatogenesis, but without the interference of a significant decline in general health due to advanced aging, we chose a mouse strain with a midlevel life expectancy. BALB/c mice have a normal life span of at least half a year beyond the end of our experiment (Van Zant \& de Haan 1999), minimizing the negative influence of poor health on testicular function. The BALB/C mice, in comparison with other readily available mouse strains, also have a mid-range bonemarrow turnover (Van Zant \& de Haan 1999). This guarantees that the bone marrow of our animals recovers quickly after cytotoxic treatment or irradiation, minimizing the effects of poor health on testicular physiology and also minimizing the number of animals lost due to health complications related to the gonadotoxic treatments. Since, in BALB/c mice, natural mortality at 1 year of age is very low but fertility potential might be declining, we assume that (a) the somatic and/or germ cells in the testis at 12 months appear normal under non-challenging conditions, but might show already age-related changes and thus might differ from the 3-month old mice after a cytotoxic challenge. This potential age-response will be detectable in the severity of spermatogenic depletion and the degree and kinetics of testicular and spermatogenic recovery. (b) That the 1-year old BALB/c mice will show an excellent health status rendering them sufficiently robust to recover from the gonadotoxic treatments.

All the procedures were in compliance and had been approved by the University of Pittsburgh Institutional Animal Care and Use Committee and animal husbandry was provided by the Division of Laboratory Animal Resources of the University of Pittsburgh School of Medicine.

\section{Gonadotoxic treatment procedures}

Within the two age groups, the animals were randomly assigned to four experimental groups (testicular cooling, Busulfan treatment, $\mathrm{X}$-irradiation, control; Table 1). For testicular cooling, the animals were anesthetized and placed on Styrofoam boards with openings $(2-3 \mathrm{~cm})$, allowing the scrotum to be suspended in ice-cold water for $30 \mathrm{~min}$. For Busulfan treatment, the animals received one single IP injection of Busulfan (Sigma \#B2635; $16 \mathrm{mg} / \mathrm{ml}$ in dimethylsulfoxide (DMSO)/saline 1:1) at $40 \mathrm{mg} / \mathrm{kg}$ bodyweight. For X-irradiation, the animals were anesthetized and the lower abdomen including the testes was irradiated with one single dose of 5 Gy using an animal irradiator containing a permanent cesium source (Gammacell 40, Atomic Energy of Canada Ltd., Kanada, Ontario, Canada). Head, thorax and upper abdomen of the animals were protected from radiation by lead shielding and received only 0.2 Gy of radiation scatter. The applied doses were monitored using Gamma and X-ray Dosimeters (\#686, Dosimeter Corporation, Cincinnati, OH, USA) during 
Table 1 Anatomical data (average \pm s.D.) for all study groups and time points.

\begin{tabular}{llll}
\hline Age/TP & $\begin{array}{l}\text { Treatment } \\
\text { (animal \#) }\end{array}$ & \multicolumn{1}{c}{ BW $(\mathrm{g})$} & \multicolumn{1}{c}{ SVW $(\mathrm{mg})$} \\
\hline Young 2 weeks & Control (7) & $27.00( \pm 0.82)$ & $391.86( \pm 86.17)$ \\
& Test. Cool. (7) & $26.00( \pm 1.15)$ & $390.71( \pm 105.02)$ \\
& Busulfan (6) & $27.33( \pm 1.63)$ & $323.17( \pm 17.00)$ \\
& X-irradiation (7) & $26.14(1.86)$ & $286.00( \pm 32.32)^{*}$ \\
Young 6 weeks & Control (7) & $28.71( \pm 1.38)$ & $416.71( \pm 74.02)$ \\
& Test. Cool. (7) & $29.14( \pm 1.35)$ & $394.43( \pm 99.63)$ \\
& Busulfan (7) & $27.92( \pm 1.11)$ & $296.43( \pm 43.55)^{*}$ \\
Old 2 weeks & X-Irradiation (8) & $30.13( \pm 1.13)$ & $355.13( \pm 77.34)$ \\
& Control (9) & $32.22( \pm 2.82)$ & $495.67( \pm 103.90)$ \\
& Test. Cool. (8) & $34.00( \pm 2.27)$ & $547.50( \pm 100.92)$ \\
& Busulfan (5) & $33.60( \pm 2.51)$ & $603.20( \pm 137.24)$ \\
Old 6 weeks & X-Irradiation (8) & $32.38( \pm 2.88)$ & $447.75( \pm 46.37)$ \\
& Control (7) & $35.29( \pm 2.29)$ & $552.29( \pm 243.61)$ \\
& Test. Cool. (7) & $36.71( \pm 2.43)$ & $620.43( \pm 150.34)$ \\
& Busulfan (6) & $36.50( \pm 2.59)$ & $452.50( \pm 55.91)$ \\
& X-Irradiation (7) & $33.71( \pm 2.87)$ & $462.86( \pm 115.78)$ \\
& & &
\end{tabular}

*Indicates a statistically significant difference from the control in the same age group and at the same time point (Kruskal-Wallis one-way ANOVA on ranks; $P<0.05)$.

each irradiation procedure. Controls were sham-irradiated, received sham testicular cooling (scrotum placed for $30 \mathrm{~min}$ in water at $35^{\circ} \mathrm{C}$; procedure as described above), and were injected with DMSO/saline (1:1) according to bodyweight.

\section{Sample retrieval}

At the pre-determined time points ( 2 and 6 weeks after (sham-) treatment), mice were killed by exsanguination via heart puncture under deep anesthesia. Body weight, seminal vesicle weight, and testis weights were recorded. Each testis was cut into four pieces of similar size, which were fixed in Bouin's fixative overnight and then transferred to $70 \%$ ethanol.

\section{Histology and evaluation}

Testis tissue was embedded in resin (Technovit 7100, Heraeus Kulzer, Hanau, Germany), sectioned to 2 and $4 \mu \mathrm{m}$ and stained with the Periodic Acid-Schiff's Reagent method followed by hematoxylin counterstaining.

The following parameters were determined for each sample:

(a) Point counting approach for the determination of the proportions of interstitium, seminiferous epithelium, and lumen: histological sections showing the largest testicular surface area of testicular tissue were observed using a primary magnification of $25 \times$. The four corners of a rectangular frame within the total field of view were employed for a point counting approach. Twenty-five not overlapping fields per animal were selected by a systematic random approach. The four reference points in each analysis field were subjected either to the interstitium, the seminiferous epithelium, or the tubule lumen. From these counts of a total of 100 points per sample, the volume density occupied by the tubule (epithelium + lumen) and the interstitial compartment were calculated as relative values. The volume fraction of each compartment (in milligram) was calculated using the point counting data in correlation to the total testis weight of each testis.

(b) Diameter of seminiferous tubules: using a micrometer scale, the smallest diameter of 25 different, randomly selected round cross-sections of seminiferous tubules were measured for each sample. The average tubule diameter for each sample was calculated as a mean of these 25 values.

(c) Length of seminiferous tubules per testis: using the average radius of the seminiferous tubules $(r)$ and the volume fraction of seminiferous tubules $(V)$ as calculated by point counting (see above), the combined length $(h)$ of seminiferous tubules for each testis was calculated using the formula $h=V / \pi * r^{2}$.

(d) Cell counts: five randomly depicted micrographs in the most widespread cross-section of a mouse testis were digitally recorded using a primary magnification of $25 \times$. Each of these micrographs represents an area of $194700 \mu \mathrm{m}^{2}$. The total numbers of Sertoli cells, A-spermatogonia, B-spermatogonia, spermatocytes, round spermatids, and elongated spermatids in each micrograph were determined, and the average number of each cell type per $194700 \mu^{2}$ was calculated (total/5). In addition, the average number of cells of each germ cell type per 100 Sertoli cells was determined. Sertoli cells were identified by their irregularly shaped nuclei, which were often positioned towards to the basement membrane and contained multiple nucleoli. Type A and B spermatogonia, spermatocytes, round and elongated spermatids were identified according to the characteristics described by Russell et al. (1990).

(e) Spermatogenic repopulation index: for each mouse, 25 randomly selected seminiferous tubules were scored to determine the presence of B-spermatogonia and more advanced germ cell types at the 6-week time-point. The data were combined for each treatment group and the average relative number of seminiferous tubules showing B-spermatogonia or more advanced germ cell types was calculated.

\section{Image acquisition and statistical analysis}

Samples were analyzed using a Nikon Eclipse E800 fluorescence microscope (Nikon, Melville, NY, USA) with attached digital camera (Olympus, Melville, NY, 
USA). All images were acquired digitally using MagnaFire Software (Optronics, Goleta, CA, USA).

Statistical analysis was performed using SigmaStat 3.1 (Systat Software, Inc., Point Richmond, CA, USA). As a post hoc test Kruskal-Wallis one-way ANOVA on ranks was used. Statistical significance was set at $P<0.05$.

\section{Results}

\section{General health and body weight}

One hundred and twenty-five mice entered the study, approximately $10 \%$ of the animals died during the study. The losses were evenly distributed in both age groups. While most of the animals in the younger group were lost as a response to the treatments, the losses of the old mice occurred randomly. However, the health status of most experimental animals throughout the study was excellent and the 113 survivors were healthy when selected for analysis of the data. Throughout the study, the older age group showed an approximately $20 \%$ higher body weight compared with the young group which is in accordance to the normal gain of body weight during that life span (Wirth-Dzieciolowska \& Czuminska 2000, Table 1). No significant differences in body weight were recorded between treatment groups in the same age group.

\section{Seminal vesicle weight}

Seminal vesicle weights of the testicular cooling groups were not different from controls. No significant changes of seminal vesicles were observed in old mice in the other two groups despite of lower levels in young mice at 2 weeks post-treatment in the X-irradiated and at 6 weeks post-treatment in the Busulfan-treated group (Table 1).

\section{Histology: qualitative aspects}

The seminiferous epithelia of the control animals of both age groups and at both time points showed undisturbed spermatogenesis and no obvious degenerative changes (Fig. 1a). A specific feature observed after testicular cooling were multinucleated germ cells which were frequently observed at both time point and in both age groups (Fig. 1b). Both, Busulfan treatment and X-irradiation induced a severe depletion of germ cells, which resulted in a high number of Sertoli cell-only tubules at the 6 weeks time point in both age groups (Fig. 1c and d).

\section{Testis weight}

In young and old mice, testis weights decreased significantly 2 and 6 weeks after X-irradiation when compared with the respective control group (Fig. 2a and b). While Busulfan had very similar effects in young mice, the decrease became only significant at 6 weeks in the older group.

\section{Results from morphometric analysis}

While no significant differences were observed in any of the analyzed parameters between the controls and testicular cooling, the volume fraction of the tubule compartment decreased significantly after Busulfan treatment at 6 weeks and after X-irradiation at both
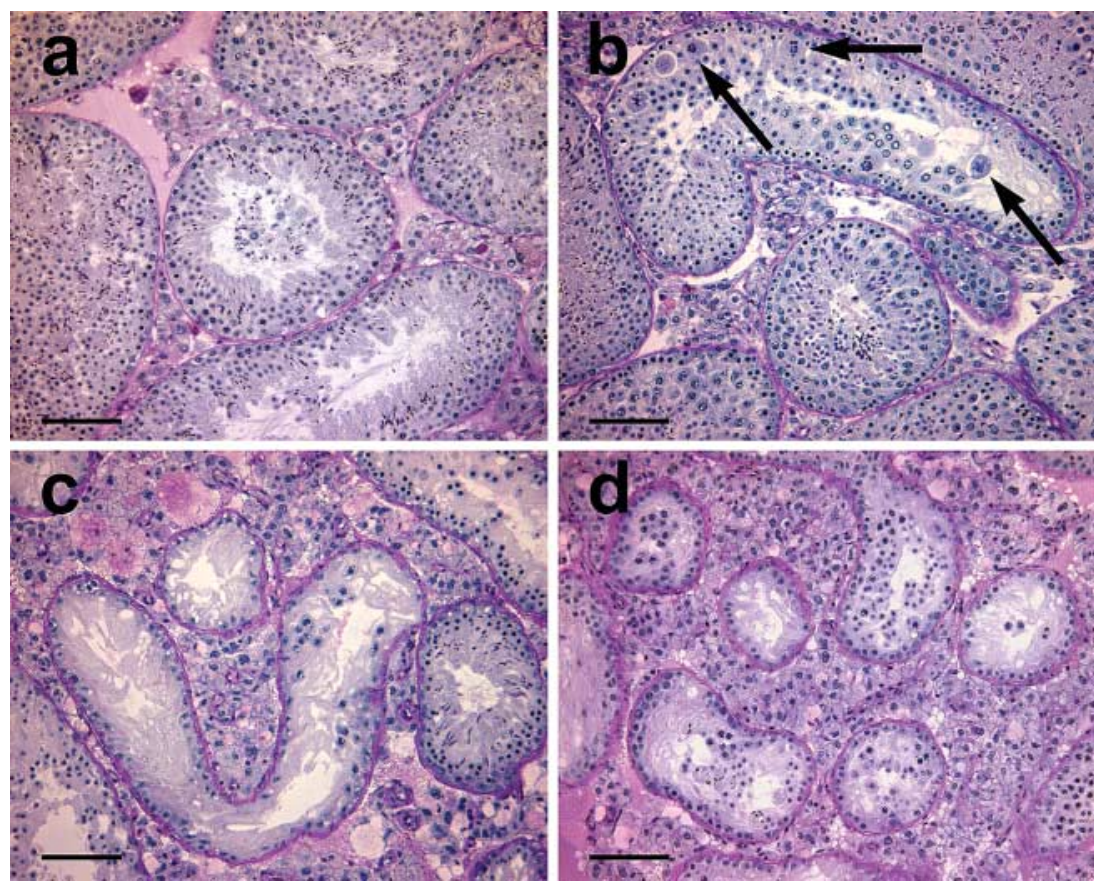

Figure 1 Histological cross-sections of testicular tissue of old mice harvested 6 weeks posttreatment. (a) Control; (b) testicular cooling; (c) Busulfan treatment; and (d) X-irradiation. Note the multinucleated cells (arrows) after testis cooling, and the Sertoli cell-only tubules after Busulfan-treatment and X-irradiation. 
(a)

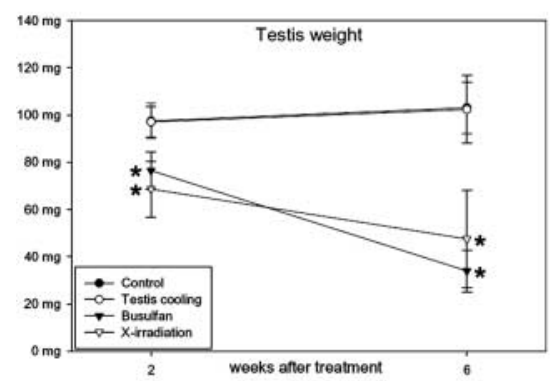

(c)

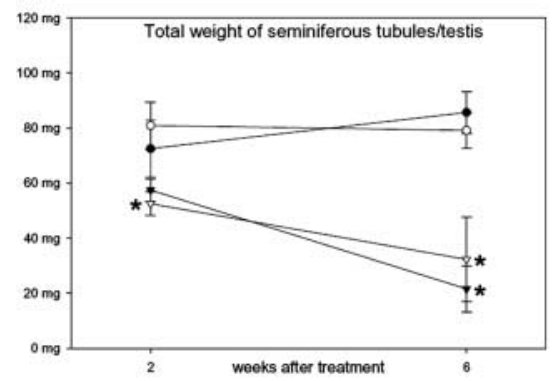

(e)

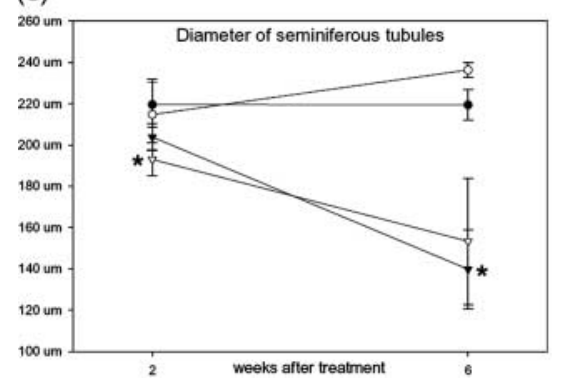

(b)

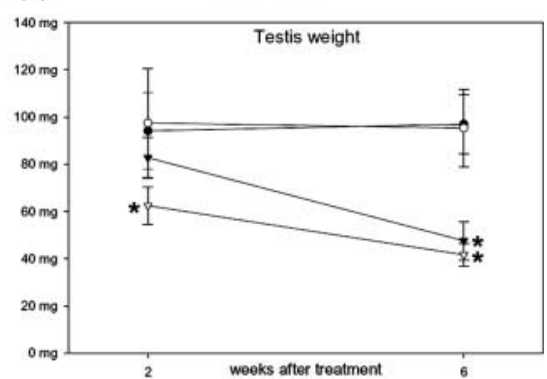

(d)

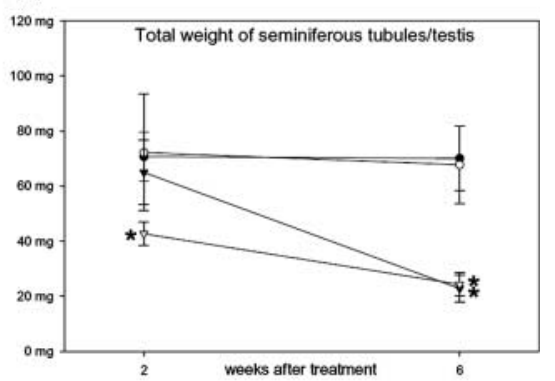

(f)

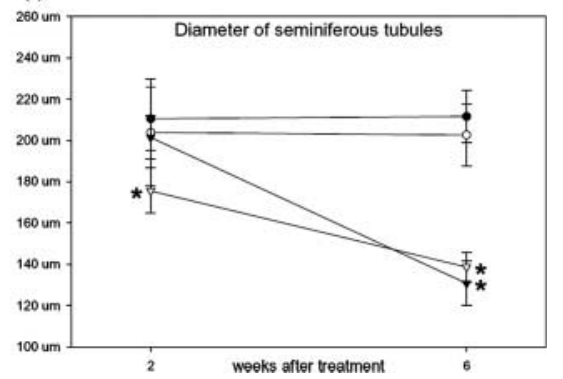

Figure 2 Testis weight $(a, b)$, total weight of the seminiferous tubules/testis (c, d), and diameter of the seminiferous tubules $(e, f ; \pm$ s.D.) of old and young mice 2 and 6 weeks after treatment. *Indicates a statistically significant difference from the control in the same age group and at the same time point (Kruskal-Wallis one-way ANOVA on ranks; $P<0.05)$. time points in both young and old mice (Fig. 2c and d). The significant differences detected in the tubule diameter mirrored the changes of volume fractions of seminiferous tubules (Fig. 2e and f). Both the parameters indicate that the testicular involution is primarily due to a reduction of the tubule compartment, which corresponds to the qualitative observation showing a proportionally larger interstitial area in Busulfan-treated and irradiated mice (Fig. 1c and d). The volume fraction of the interstitial compartment ranged from 12.37 ( \pm 3.32) mg (young mice 6 weeks after Busulfan treatment) to 27.56 ( \pm 12.95$) \mathrm{mg}$ (old mice 6 weeks after testicular cooling). At both time points and in both age groups, the differences in interstitial volume fraction between the corresponding control and any treatment group did not reach statistical significance confirming that Busulfan treatment and irradiation evoke no significant effect on volume density of the interstitial compartment. The length of seminiferous tubules as calculated per testis varied between $1.36( \pm 0.18) \mathrm{mm}$ (young mice 6 weeks after Busulfan treatment) and $2.27( \pm 0.11) \mathrm{mm}$ (young control mice at the 6 weeks time point). Tubule length did not differ significantly from the respective controls in any of the age and treatment groups indicating in conjunction with the significant effects on tubule diameter that the involution of the tubule compartment is primarily due to a thinning of the seminiferous tubules and not due to a reduction in length.

\section{Cell counts}

Numbers of A-spermatogonia, B-spermatogonia, spermatocytes, round spermatids and elongated spermatids were calculated per 100 Sertoli cells and are plotted in Figs 3 and 4. No changes were noted to occur in the testicular cooling group at any time point in both age groups. In contrast, germ cell numbers in Busulfantreated and irradiated mice showed the expected successive depletion of germ cells as both treatments affect most strongly the pre-meiotic germ cells, but to a lesser degree the meiotic and post-meiotic germ cells leading to a depletional wave (van Keulen \& deRooij 1975, Meistrich et al. 1978). Overall, at 2 weeks, spermatocytes show a significant depletion in both treatment and age groups and the depletion of germ cells is more advanced at 6 weeks when all meiotic and post-meiotic germ cells are depleted (Figs 3 and 4). When looked upon in more detail, the X-irradiated, but 


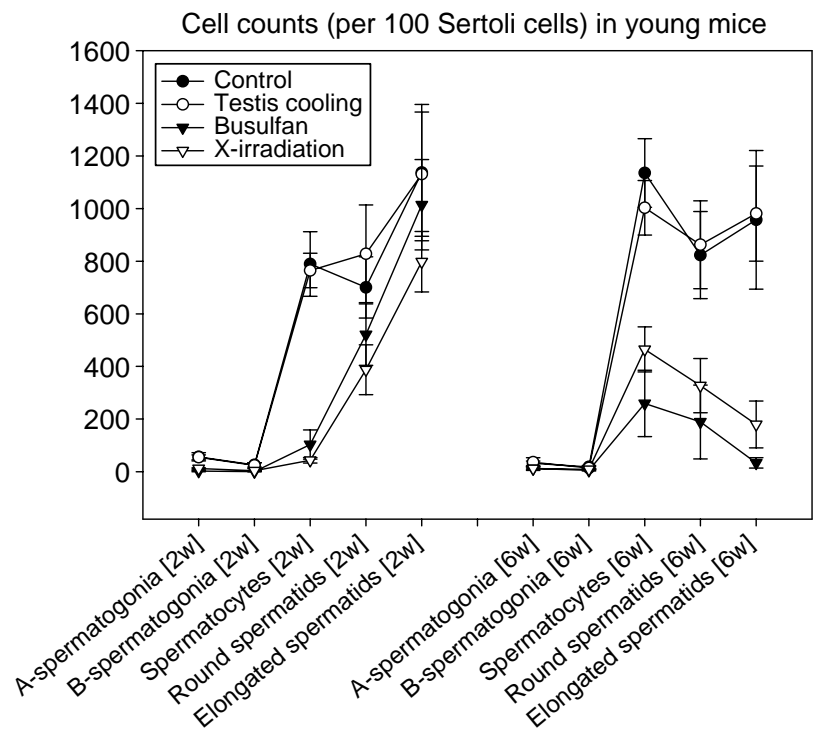

Figure 3 Cell counts $( \pm$ S.E.) in young mice 2 weeks $(2 w)$ and 6 weeks (6w) after treatment.

not the Busulfan-treated young and old mice showed a significant reduction of round spermatids already at 2 weeks when compared with controls. Six weeks after treatment, spermatocytes in young and old mice of both the X-irradiated and the Busulfan-treated groups were significantly reduced when compared with the respective controls, but had significantly recovered when compared with the counts of the same age/treatment groups at the 2-week time-point. The cell counts for both age groups were nearly identical. The only significant differences were recorded at 6 weeks in irradiated mice when the numbers of spermatocytes and round spermatids in the young animals were higher than in old animals.

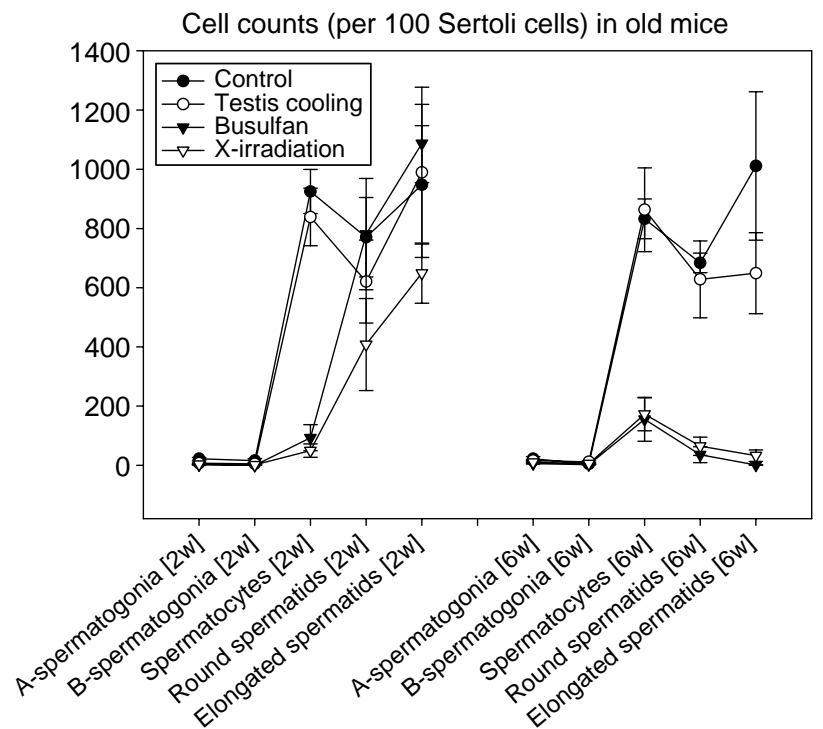

Figure 4 Cell counts $( \pm$ s.E. $)$ in old mice 2 weeks $(2 w)$ and 6 weeks $(6 w)$ after treatment.

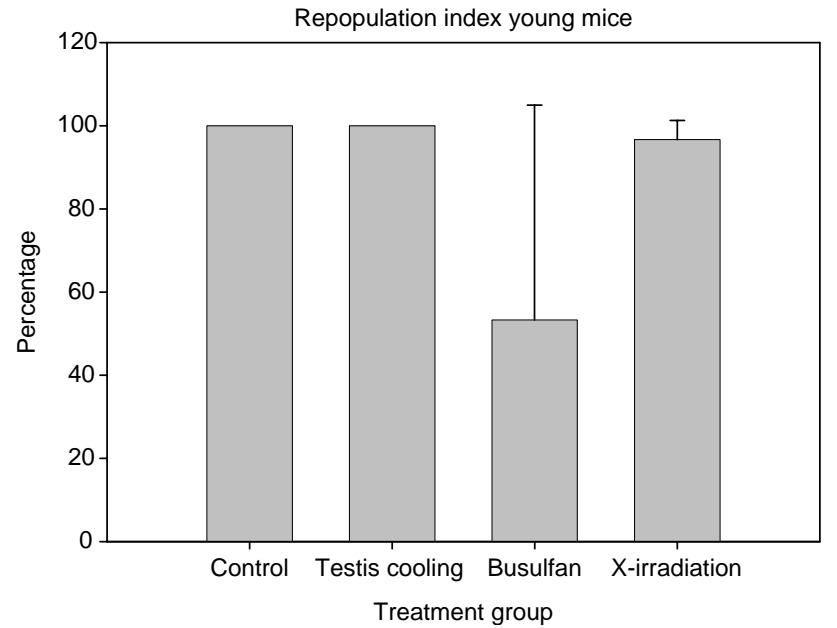

Figure 5 Repopulation indices ( \pm s.D.) in young mice as evaluated 6 weeks post-treatment. None of the treatment groups differed significantly from the control group.

In addition, the repopulation index (relative number of seminiferous tubules with recolonizing B-spermatogonia or more mature germ cells) estimated at 6 weeks posttreatment were lower in the Busulfan-treated animals when compared with the other treatments in both age groups, but reached only significant levels in the old mice (Figs 5 and 6). The overall high repopulation indices indicate that spermatogenic recovery is expected to proceed in all treatment groups at both ages at later time points.

\section{Discussion}

Our study reveals with a new approach that testicular function at the end of the normal fertile period of mice is not associated with increased vulnerability to gonadotoxic insults. Neither testicular cooling, exposure to ionizing radiation nor exposure to Busulfan revealed a

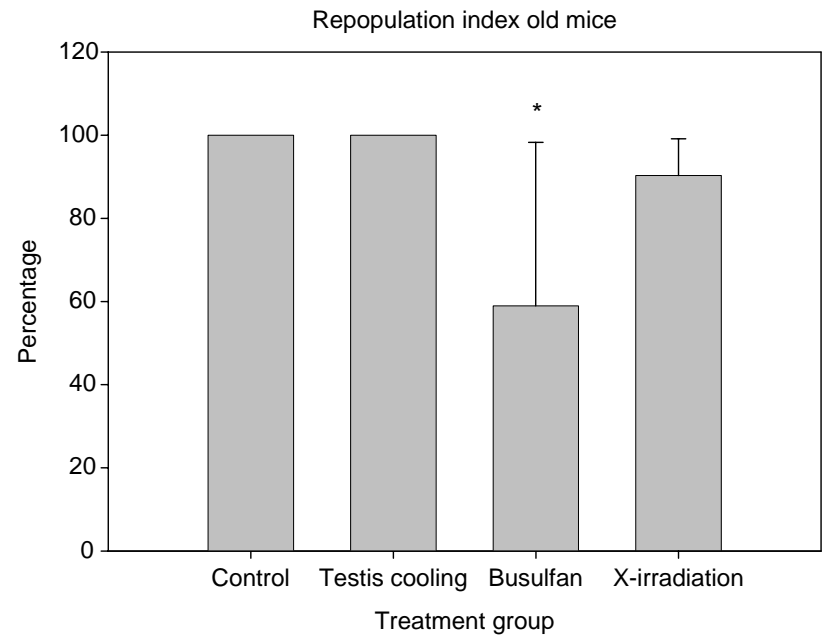

Figure 6 Repopulation indices ( \pm s.D.) in old mice as evaluated 6 weeks post-treatment. Note that the Busulfan-treated group differed significantly from the control group $\left(^{*}\right)$. 
more intense age-related response on the depletion and recovery of spermatogenesis and the extent of repopulation occurring from remaining spermatogonial stem cells.

In rats, testicular cooling has been shown to affect the somatic environment and specifically the Sertoli cells, inducing a severe depletion of spermatogenesis (Zhang et al. 2004). The authors of this study stated that this method exerts its effect by providing a disturbed microenvironment unable to sustain spermatogenesis rather than by specifically targeting the germ cells. In contrast to our expectations, cooling had no severe detrimental effects on spermatogenesis in our study. It only induced a not age-related increase of multinucleated germ cells. Although these multinucleated cells were abundant in the acute response to cooling, neither the gross testis weights nor any of the quantitative parameters were different from control testes in both the age groups. In contrast to the earlier study performed on rats (Zhang et al. 2004), the effect of testicular cooling observed here in mice was thus both much milder and germ cell specific. This much more attenuated response to testicular cooling could be both due to speciesspecific differences or to a difference in the study design, as in our study, the testes remained in the scrotum whereas in the rat study (Zhang et al. 2004), the testes were dissected and exposed to cold externally.

The two alternative strategies, i.e. exposure to ionizing radiation and Busulfan treatment have been well studied previously and both are known to primarily affect the germ cell compartment (van Keulen \& deRooij 1975, Meistrich et al. 1978, Meistrich 1993). It is well documented that a balanced dose of Busulfan (van Keulen \& deRooij 1975, Brinster \& Zimmermann 1994) or X-irradiation (Meistrich et al. 1978) results in a complete loss of germ cells including the majority of the spermatogonial stem cells in animal models without exerting devastating defects on the somatic environment since transplantation of stem cells re-induced spermatogenesis (Brinster \& Zimmermann 1994). The doses of Busulfan $(40 \mathrm{mg} / \mathrm{kg}$ ) and radiation (5 Gy) were previously shown to achieve full depletion of germ cells, but only partial depletion of testicular stem cells to allow spontaneous recovery to occur (Meistrich et al. 1978, Brinster \& Zimmermann 1994). We obtained the expected responses from both treatments, leading to involution of the testes due to a depletional wave being incomplete at 2 weeks post-treatment and a continued loss of germ cells until week 6 .

Although significant differences were observed at some points, the seminal vesicle weight reveals that the androgen status was only marginally affected, as even the lowest detected levels were 10-fold higher than the seminal vesicle weight in castrate mice $(<20 \mathrm{mg})$. The normal androgen status and the extent of spermatogenetic depletion indicate that both treatments target specifically the germ cell, but not the somatic cells.
Considering both treatments to be germ cell specific, we interpret any difference in the depletion of spermatogenesis between the two age groups as an age-related change in the germinal compartment and any change in the extent and speed of recovery as an age-dependent change in the functionality of spermatogonial stem cells.

Both the qualitative and the quantitative testicular parameters after X-irradiation and Busulfan-treatment differ from the respective controls. The extent of reduction of tubule diameter, testis weight and volume density of seminiferous tubules was identical in the $\mathrm{X}$-irradiated and the Busulfan-treated mice within both age groups, indicating that the dose of 5 Gy for $\mathrm{X}$-irradiation and $40 \mathrm{mg}$ for Busulfan evoked comparable gonadotoxic effects. None of the treatments was followed by a detectable decrease of the volume density of the interstitium or the length of seminiferous tubules. We therefore conclude that the reduction in testis weight is related primarily to a depletion of germ cells as seminiferous tubule diameters, but not their length decrease. Sertoli and interstitial cells were neither affected by X-irradiation nor by Busulfan exposure. Since the changes observed after Busulfan treatment and $\mathrm{X}$-irradiation were similar in both age groups, we conclude that the vulnerability of the germ cells does not show an age-related change from the age of 3 months until 1 year.

At the sixth week, both young and old X-irradiated and Busulfan-treated mice show a high spermatogenic repopulation index as most seminiferous tubules contained at least few B-spermatogonia as most advanced germ cell types. A return of B-spermatogonia is a first sign of recovery and indicates that the doses used will not lead to permanent infertility but that recovery of spermatogenesis has been initiated in both age groups. At the selected doses, the kinetics of recovery appear faster after X-irradiation when compared with Busulfan treatment in both age groups. The significantly higher numbers of spermatocytes and round spermatids as well as the higher number of recolonized tubules (leading to a insignificant change of the repopulation index) in younger animals at the 6 weeks time-point indicate a faster recovery of the young age group when compared with older mice. However, many of the analyzed parameters show great variability, and the lack of later time points make these assumptions slightly speculative.

Recent data revealed interesting aging effects on spermatogonial stem cells and let us speculate why our study revealed surprisingly minor effects of aging during a rather substantial period of the reproductive life span of mice. Applying serial germ cell transplantation of aged mouse germ cells into younger recipients revealed that spermatogonial stem cell self-renewal continues much beyond the individuals' life span (Ryu et al. 2006). The authors conclude that deterioration of the stem cell niche and loss of appropriate balance of self-renewal and differentiation are responsible for an age-related decline 
of fertility in older mice. Another recent study compares the functional parameters of spermatogonial stem cells in young and old ROSA2 6 mice showing that the initial defects of age-related testicular regression in 1-year old mice are independent from the stem cells since their colonization potential is similar to that of younger mice (Zhang et al. 2006). However, spermatogonial stem cells from 2-year old mice show reduced functionality. The authors conclude that the spermatogonial stem cell and their somatic environment are involved in the agerelated decline of spermatogenesis in mice. These studies reveal that the cause for age-related decline of spermatogenesis is primarily provoked by the deterioration of the somatic environment. The slowly, if at all, aging spermatogonial stem cells are highly selected throughout the reproductive life but continue to recolonize and generate differentiating progeny even long time after the normal reproductive life span. In respect to our data, we can conclude that the somatic environment in 1-year old mice is not more sensitive to cooling, irradiation and cytotoxic injury. While irradiation and Busulfan treatment affect mainly the germ cell compartment, the old mouse testis is capable of recolonizing the non-affected somatic environment from the remaining stem cells. We interpret the inability to detect an age-dependent effect of cooling in our study by the overall mild defect which our experimental strategy induced. Future studies should focus on a more significant deterioration of the somatic environment to test from which age onwards the somatic environment becomes more vulnerable to injury.

In conclusion, testes from 3-month and 1-year old mice respond similarly to gonadal injury and recover with comparable kinetics from germ cell depletion via X-irradiation or Busulfan exposure. Testicular cooling exerted no severe damage, but specifically induced multinucleation of germ cells. We conclude that the testis in 1-year old mice contains a healthy complement of stem cells capable to induce spermatogenic recovery after injury and that the aging testicular microenvironment is not more vulnerable in response to irradiation, Busulfan-treatment or testicular cooling.

\section{Acknowledgements}

Grant support: This work was financially supported by startup funds from the University of Pittsburgh School of Medicine and $\mathrm{NIH}$ grant 5R21 AG024914-02. The authors declare that there is no conflict of interest that would prejudice the impartiality of this scientific work.

\section{References}

Brinkworth MH, Weinbauer GF, Bergmann M \& Nieschlag E 1997 Apoptosis as a mechanism of germ cell loss in elderly men. International Journal of Andrology 20 222-228.
Brinster RL \& Zimmermann JW 1994 Spermatogenesis following male germ-cell transplantation. PNAS 91 11287-11302.

de Miguel MP, Bethencourt FR, Arenas MI, Fraile B \& Paniagua R 1997 Intermediate filaments in the Sertoli cells of the ageing human testis. Virchows Archiv 431 131-138.

Ehmcke J, Wistuba J \& Schlatt S 2006 Spermatogonial stem cells: questions, models and perspectives. Human Reproduction Update 12 275-282.

Eskenazi B, Wyrobek AJ, Sloter E, Kidd SA, Moore L, Young S \& Moore D 2003 The association of age and semen quality in healthy men. Human Reproduction 18 447-454.

Fauser BCJM 2000 Follicle pool depletion: factors involved and implication. Fertility and Sterility 74 629-630.

Gosden RG \& Faddy MJ 1994 Ovarian aging, follicular depletion, and steroidogenesis. Experimental Gerontology 29 265-274.

Haidl G, Jung A \& Schill WB 1996 Ageing and sperm function. Human Reproduction 11 558-560.

Honore LH 1978 Ageing changes in the human testis: a light microscopic study. Gerontology 24 58-65.

Johnson L 1989 Evaluation of the human testis and its age-related dysfunction. Progress in Clinical and Biological Research 302 $35-67$.

Johnson L, Petty CS \& Neaves WB 1986 Age-related variation in seminiferous tubules in men. A stereologic evaluation. Journal of Andrology 7 316-322.

Kimura M, Itoh N, Takagi S, Sasao T, Takahashi A, Masumori N \& Tsukamoto T 2003 Balance of apoptosis and proliferation of germ cells related to spermatogenesis in aged men. Journal of Andrology 24 185-191.

Meistrich ML 1993 Effects of chemotherapy and radiotherapy on spermatogenesis. European Urology 23 136-141.

Meistrich ML, Hunter NR, Suzuki N, Trostle PK \& Withers HR 1978 Gradual regeneration of mouse testicular stem cells after exposure to ionizing radiation. Radiation Research 74 349-362.

Miething A 2005 Arrested germ cell divisions in the ageing human testis. Andrologia 37 10-16.

Neaves WB, Johnson L, Porter JC, Parker CR Jr \& Petty CS 1984 Leydig cell numbers, daily sperm production, and serum gonadotropin levels in aging men. Journal of Clinical Endocrinology and Metabolism 59 756-763.

Nieschlag E, Lammers U, Freischem CW, Langer K \& Wickings EJ 1982 Reproductive functions in young fathers and grandfathers. Journal of Clinical Endocrinology and Metabolism 55 676-681.

Pal L \& Santoro N 2003 Age-related decline in fertility. Endocrinology and Metabolism Clinics of North America 32 669-688.

Paniagua R, Nistal M, Amat P, Rodriguez MC \& Martin A 1987 Seminiferous tubule involution in elderly men. Biology of Reproduction 36 939-947.

Pasqualotto FF, Sobreiro BP, Hallak J, Pasqualotto EB \& Lucon AM 2005 Sperm concentration and normal sperm morphology decrease and follicle-stimulating hormone level increases with age. BJU International 96 1087-1091.

Plas E, Berger P, Hermann M \& Plueger H 2000 Effects of aging on male fertility? Experimental Gerontology 35 543-551.

Radford J 2003 Restoration of fertility after treatment for cancer. Hormone Research 59 21-23.

Russell LD, Ettlin RA, Sinha Hikim AP \& Clegg ED 1990 In Histological and Histopathological Evaluation of the Testis, Ed. LD Russell. Clearwater, Florida: Cache River Press.

Ryu BY, Orwig KE, Oatley JM, Avarbock MR \& Brinster RL 2006 Effects of aging and niche microenvironment on spermatogonial stem cell self-renewal. Stem Cells 24 1505-1511.

Schlatt S, von Schoenfeldt V \& Schepers AG 2000 Male germ cell transplantation: an experimental approach with a clinical perspective. British Medical Bulletin 56 824-836.

Skinner MK 2005 Regulation of primordial follicle assembly and development. Human Reproduction Update 11 461-471. 
Suzuki N \& Withers HR 1978 Exponential decrease during aging and random lifetime of mouse spermatogonial stem cells. Science $\mathbf{2 0 2}$ $1214-1215$.

Takagi S, Itoh N, Kimura M, Sasao T \& Tsukamoto T 2001 Spermatogonial proliferation and apoptosis in hypospermatogenesis associated with nonobstructive azoospermia. Fertility and Sterility 76 901-907.

Van Keulen CJ \& deRooij DG 1975 Spermatogenic clones developing from repopulating stem cells surviving a high dose of an alkylating agent. Cell and Tissue Kinetics 8 543-551.

Van Zant G \& de Haan G 1999 Genetic control of lifespan: studies from animal models. Expert Reviews in Molecular Medicine 1 1-12.

Wallace WH \& Thompson AB 2003 Preservation of fertility in children treated for cancer. Archives of Disease in Childhood 88 493-496.

Wirth-Dzieciolowska E \& Czuminska K 2000 Longevity and aging of mice from lines divergently selected for body weight for over 90 generations. Biogerontology 1 171-178.
Yakirevich E, Sabo E, Dirnfeld M, Sova Y, Spagnoli GC \& Resnick MB 2003 Morphometrical quantification of spermatogonial germ cells with the 57B anti-MAGE-A4 antibody in the evaluation of testicular biopsies for azoospermia. Applied Immunohistochemistry \& Molecular Morphology 11 37-44.

Zhang Z, Short RV, Meehan T, deKretser DM, Renfree MB \& Loveland KL 2004 Functional analysis of the cooled rat testis. Journal of Andrology 25 57-68.

Zhang X, Ebata KT, Robaire B \& Nagano MC 2006 Aging of male germ line stem cells in mice. Biology of Reproduction 74 119-124.

Received 14 August 2006

First decision 11 September 2006

Accepted 23 October 2006 